\title{
Coherent states for the bouncing pendulum and the paddle ball
}

\author{
Mark Andrews ${ }^{\text {a) }}$ \\ Department of Physics and Theoretical Physics, Australian National University, ACT 0200, Australia
}

(Received 8 February 2007; accepted 22 November 2007)

\begin{abstract}
The coherent states of the simple harmonic oscillator with an impenetrable barrier at its center are studied. This half oscillator is the equivalent of a pendulum that bounces elastically off a vertical wall directly below the point of suspension with the angle of swing sufficiently small. The system can also be considered as a paddle ball, where the paddle is fixed and the ball is constrained by a spring attached to the paddle. The coherent states are almost the same as the familiar Gaussian coherent states of the full oscillator, except when they overlap the barrier. The solutions can be easily extended to two and three dimensions and gravity can be included if the impenetrable barrier is vertical. To better understand the form of the expectation values of the position and momentum, we investigate some general aspects of the effect of impenetrable barriers on the dynamics of wavepackets. (C) 2008 American Association of Physics Teachers.
\end{abstract}

[DOI: 10.1119/1.2825388]

\section{INTRODUCTION}

Many textbooks ${ }^{1,2}$ and articles ${ }^{3,4}$ on quantum mechanics discuss the coherent states of the simple harmonic oscillator in one dimension. Some authors ${ }^{5,6}$ mention the halfoscillator, for which there is an impenetrable barrier at the potential minimum (here taken to be at $x=0$ ), but usually just mention that its energy eigenstates are the odd eigenstates of the full oscillator. ${ }^{7}$

The half oscillator can represent a pendulum bouncing elastically off a vertical wall directly below the point of suspension with the angle of swing sufficiently small. It can also be considered as a paddle ball, ${ }^{5}$ where the paddle is fixed and the ball is constrained by a spring attached to the paddle. For the pendulum, gravity is necessary; in the simplest model of the paddle ball, gravity is neglected.

The coherent states of the half oscillator are of interest because they are mathematically simple (involving only Gaussians) and, while remaining as narrowly spread in position and momentum as possible, they closely follow the classical motion.

\section{COHERENT STATES OF THE FULL OSCILLATOR}

There are many different ways to approach coherent states. ${ }^{1,2}$ We give one approach in Appendix A. Here, all we need is that the coherent state can be taken to be

$$
\psi(x, t)=N_{0}^{-1 / 2} \exp \left[-i \theta(t)+i \bar{p} x / \hbar-(x-\bar{x})^{2} / 4 d^{2}\right],
$$

where $\bar{x}$ and $\bar{p}$ are the expectation values of position and momentum, respectively, $m$ is the mass of the ball or pendulum bob, and $\omega$ is the angular frequency of the oscillation with $d=\sqrt{\hbar /(2 m \omega)}$. From Ehrenfest's theorem, $\bar{x}$ and $\bar{p}$ must follow a classical trajectory, and we take $\bar{x}=a \cos \omega t$ and $\bar{p}=-m \omega a \sin \omega t$. The time-dependent phase is $\theta(t)=\omega t / 2+\bar{p} \bar{x} / 2 \hbar$, and the normalizing factor is $N_{0}$ $=\int_{-\infty}^{\infty} \exp \left(-x^{2} / 2 d^{2}\right) d x=d \sqrt{2 \pi}$.

The special property of these coherent states is that they remain Gaussian and that the spreads in position $\Delta x$ $\left.=\left\langle(x-\bar{x})^{2}\right)\right\rangle^{1 / 2}=d$ and momentum $\left.\Delta p=\left\langle(p-\bar{p})^{2}\right)\right\rangle^{1 / 2}=\hbar / 2 d$ are constant and have the minimum uncertainty product $\Delta x \Delta p=\hbar / 2$. As discussed in Appendix A, every state with the minimum uncertainty product must have the form given in Eq. (1), but the coherent states of the oscillator require that $d$ take the value $\sqrt{\hbar /(2 m \omega)}$.

\section{COHERENT STATES OF THE HALF OSCILLATOR}

The antisymmetric wavefunction

$$
\phi(x, t)=N_{1}^{-1 / 2}[\psi(x, t)-\psi(-x, t)],
$$

is a solution of Schrödinger's equation for the oscillator for $x>0$ and satisfies the required boundary condition that $\phi(x, t) \rightarrow 0$ as $x \rightarrow 0$. Therefore, $\phi(x, t)$ is a solution of Schrödinger's equation for the half oscillator. The normalization of $\phi(x, t)$ is shown in Appendix $\mathrm{B}$ to be $N_{1}$ $=\left(1-e^{-a^{2} / 2 d^{2}}\right)$. To understand the wavefunction $\phi(x, t)$, it is useful to view it in the whole $x$-space, where it is the difference of two Gaussian packets oscillating with the same classical amplitude but $180^{\circ}$ out of phase. Apart from the time interval during which these packets overlap (and interfere), only one is in the physical region $x>0$, and this packet is then an almost exact approximation to $\phi(x, t)$ because the exponential tail of the other Gaussian packet is negligible. When a packet in the physical region returns to overlap the barrier, it interferes with the other packet, which then emerges as the physical one. This behavior is essentially the same as has been described for a free wavepacket bouncing off an impenetrable barrier, ${ }^{8}$ except that in the latter case there is no repetition of the bouncing. Figure 1 shows a sequence of plots of the magnitude of the wavefunction as it approaches the barrier.

The state $\phi$ is as coherent a state as can be found for the half oscillator. It is almost exactly a Gaussian of the same spread in position and momentum as for the coherent states of the full oscillator, except when it overlaps the barrier. During this time it has a smaller spread in position (because it overlaps its reflected part) but a much larger spread in momentum (because it is a superposition of one part moving with speed $\omega a$ in one direction and the other part moving at the same speed in the opposite direction. Figure 2 shows how these spreads behave during the bounce. 


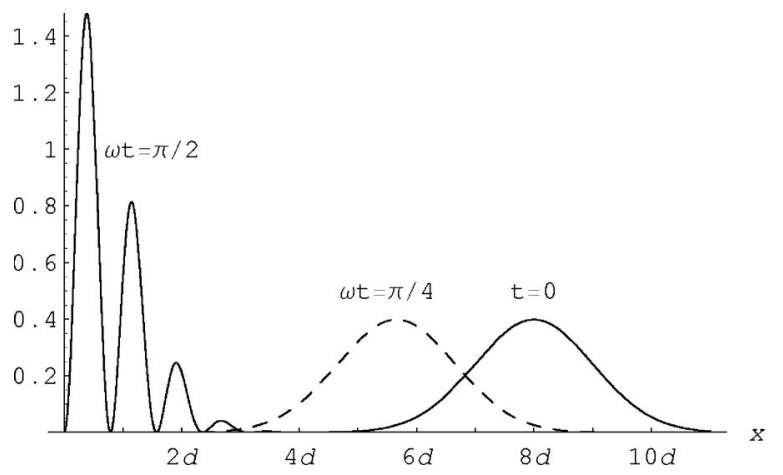

Fig. 1. Graph of $|\psi|^{2}$ at three equally spaced times for the half oscillator with amplitude $a=8 d$. At time $t=0$, the center of the wavepacket is at rest at $x$ $=8 d$. For $\omega t=\pi / 2$, the wavepacket maximally interferes with its reflected part. The units for $|\psi|^{2}$ are $1 / d$.

\section{EXTENSION TO TWO OR THREE DIMENSIONS}

The pendulum can easily be extended to two dimensions and is then referred to as the conical pendulum. In the small angle approximation, the pendulum can be modeled by an isotropic two-dimensional harmonic oscillator. The classical orbit, and the orbit of the centroid of any quantum wavepacket, is an ellipse. Because the Hamiltonian is the sum of two commuting terms, each involving only one Cartesian coordinate, an appropriate coherent state for the full oscillator can be written as a product of two one-dimensional coherent states:
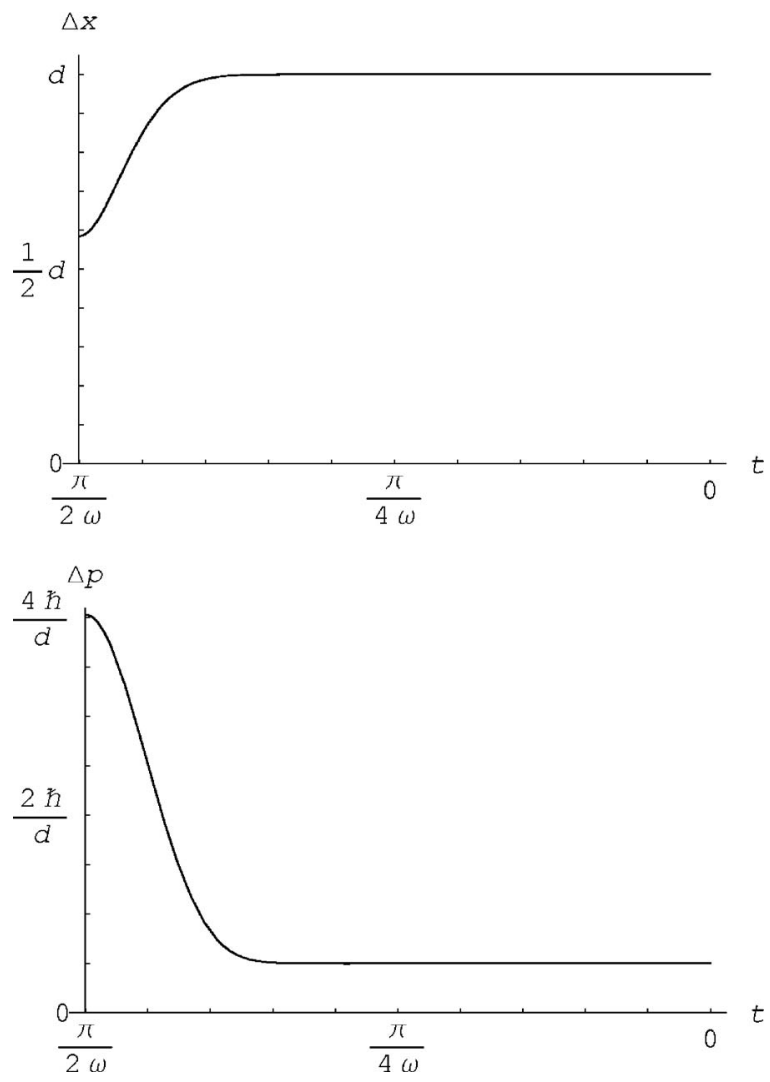

Fig. 2. The spreads $\Delta x$ and $\Delta p$ of the coherent state of the half oscillator with amplitude $a=8 d$ for one quarter of a cycle. Except for the time where the wavepacket overlaps the barrier, these spreads almost exactly equal the minimum uncertainty values of $\Delta x=d$ and $\Delta p=\hbar / 2 d$.

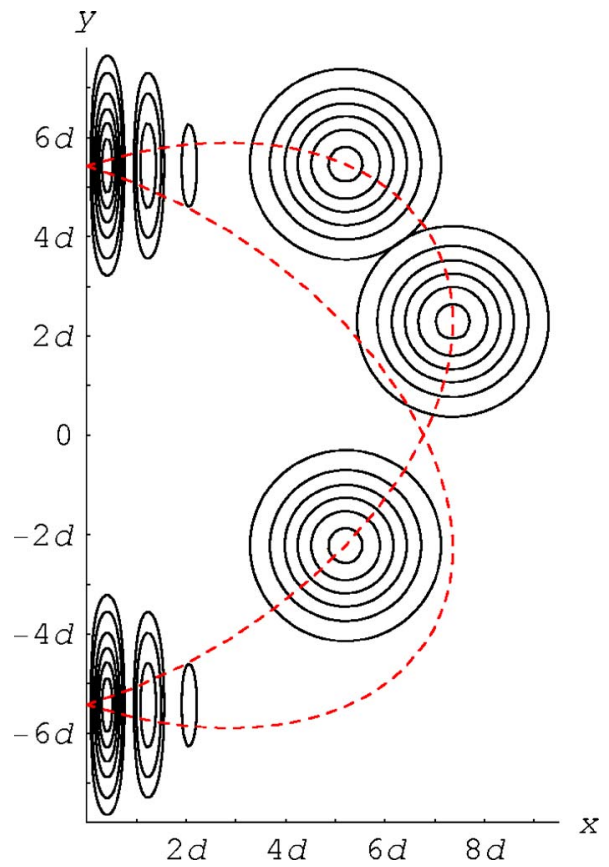

Fig. 3. Contours of $|\phi|^{2}$ at five equally spaced times (spacing $\pi / 4 \omega$ ) on one half-cycle of the two-dimensional half oscillator. The elliptical orbit has $a$ $=8 d, b=5 d$, and $\gamma=\pi / 6$. The contour interval is $0.025 / d^{2}$ for the three times when there is negligible overlap with the barrier, and $0.05 / d^{2}$ for the two times when there is maximal overlap and the interference produces a maximum that is about four times larger. The dashed lines show the complete classical trajectory. Contours of $|\phi|^{2}$ are given only for times on one half of this trajectory (though for the two bounce times, the contours apply equally to either half of the trajectory).

$$
\begin{aligned}
\psi(x, y, t)= & N_{0}^{-1} \exp \left[-i \theta(t)+i\left(\bar{p}_{x} x+\bar{p}_{y} y\right) / \hbar\right. \\
& \left.-\left[(x-\bar{x})^{2}+(y-\bar{y})^{2}\right] / 4 d^{2}\right] \\
= & N_{0}^{-1} \exp \left[-i \theta(t)+i \overline{\mathbf{p}} \cdot \mathbf{r} / \hbar-(\mathbf{r}-\overline{\mathbf{r}})^{2} / 4 d^{2}\right],
\end{aligned}
$$

where $\overline{\mathbf{r}}$ and $\overline{\mathbf{p}}$ are the expectation values of the position and momentum, respectively, which follow a classical elliptical orbit, and $\theta(t)=\omega t+\overline{\mathbf{p}} \cdot \overline{\mathbf{r}} / 2 \hbar$. In a coordinate system $x^{\prime}, y^{\prime}$ that is aligned with the axes of the ellipse, we can take $\bar{x}^{\prime}$ $=a \cos \omega t$ and $\bar{y}^{\prime}=b \sin \omega t$. If we insert the barrier, the orbit of the pendulum will, in general, be an ellipse with no axis parallel to the barrier. Therefore, we take the axes $x, y$ to be at an angle $\gamma$ to those of the ellipse and the orbit becomes $\bar{x}=a \cos \omega t \cos \gamma-b \sin \omega t \sin \gamma, \bar{y}=a \cos \omega t \sin \gamma$ $+b \sin \omega t \cos \gamma$, with the barrier at $x=0$. The part of the ellipse with $x<0$ is folded into the physical region $x>0$ via $x \rightarrow-x$, and the coherent state wavefunction is

$$
\phi(x, y, t)=N_{\gamma}^{-1 / 2}[\psi(x, y, t)-\psi(-x, y, t)] .
$$

In this case the normalizing factor is $N_{\gamma}=1-\exp \left[-\left(a^{2}+b^{2}\right.\right.$ $\left.\left.+\left(a^{2}-b^{2}\right) \cos 2 \gamma\right) / 4 d^{2}\right]$. A typical orbit and some views of the associated wavefunction are shown in Fig. 3.

The paddle ball is taken to be a fixed plane impenetrable barrier with a ball constrained by a spring attached to a point on the paddle. The pendulum is by its nature confined to two dimensions, but the paddle ball naturally extends to three. If we ignore gravity, the force on the ball is directed back to a fixed point on the paddle, and therefore the classical motion of the ball lies in a plane, which we are free to take to be the 
$(x, y)$-plane. The $z$-dependence of the wavefunction is easily incorporated by multiplying by a stationary one-dimensional Gaussian: $\phi(x, y, z, t)=N_{0}^{-1 / 2} \exp \left[-i \omega t / 2-z^{2} / 2 d^{2}\right] \phi(x, y, t)$.

\section{ADDING GRAVITY TO THE PADDLE BALL}

It is not difficult to add gravity to the paddle ball provided the paddle is vertical. Again take the barrier (that is, the paddle) to be the plane $x=0$ and the $z$-axis to point vertically upward. Then gravity requires a term $m g z$ to be added to the Hamiltonian. The coordinate $z$ appears only in the terms $m \omega^{2} z^{2} / 2+m g z$, which can be rewritten as $m \omega^{2} Z^{2} / 2-C$, where $Z=\left(z+g / \omega^{2}\right)$ and $C=m g^{2} / 2 \omega^{2}$. This added constant adds to the energy and can be ignored here. We replace the variable $z$ by $Z$ and because $p_{Z}=p_{z}$, the solution with gravity is obtained from the previous solution by replacing $z$ by $Z$. Hence, the orbits are lowered by the distance $g / \omega^{2}$. The distance $g / \omega^{2}$ is also the stretch of the spring due to gravity when the ball just hangs at rest.

If the paddle were not vertical, the solution would be much more complicated. It would not be obtained from the full oscillator solution by subtracting the solution reflected in the barrier, because the potential would no longer be symmetric under this reflection. Even dealing with gravity alone in this situation (leaving out the spring), sometimes referred to as the quantum bouncer, leads to these complications. ${ }^{9,10}$

\section{CONCLUSION}

The half oscillator, with its physical manifestations as a bouncing pendulum and a paddle ball, provides a system for which it is easy to find coherent states that are as compact as possible and follow the classical trajectories fairly closely. Apart from the short time that the wavepackets are bouncing off the barrier, they are approximately simple Gaussian wavepackets and the behavior during the bounce can be understood qualitatively. At this level, these coherent states are suitable for discussion in an introductory course on quantum mechanics as a simple extension to the standard coherent states of the oscillator. A more detailed examination of the system (in particular calculating the expectation values of the position and momentum and their squares) reveals interesting aspects of the dynamics of particles interacting with impenetrable barriers (see Appendix B). This extension could be a challenging problem for capable students.

\section{APPENDIX A: MINIMUM-UNCERTAINTY WAVEPACKETS}

The theory that is presented in most texts ${ }^{11}$ leads to the inequality $\Delta_{x} \Delta_{p} \geq \hbar / 2$ for any state $\psi$, where $\Delta_{x}^{2}=\left\langle(\hat{x}-\bar{x})^{2}\right\rangle$ with $\bar{x}=\langle\hat{x}\rangle$ and $\Delta_{p}^{2}=\left\langle(\hat{p}-\bar{p})^{2}\right\rangle$ with $\bar{p}=\langle\hat{p}\rangle$. Equality will occur only if $\psi$ is an eigenfunction of the operator $\hat{a}$ with

$$
\hat{a}=\hat{x} / 2 \Delta_{x}+i \Delta_{x} \hat{p} / \hbar
$$

where $\left[\hat{a}, \hat{a}^{\dagger}\right]=\hat{1}$. The eigenvalue must be $\alpha=\langle\hat{a}\rangle=\bar{x} / 2 \Delta_{x}$ $+i \Delta_{x} \bar{p} / \hbar$. The solution of the eigenvalue equation $\hat{a} \psi=\alpha \psi$ in Schrödinger's representation shows that, for any Hamiltonian, the wavefunction with the minimum uncertainty product $\Delta_{x} \Delta_{p}=\hbar / 2$ is

$$
\psi(x)=\left(2 \pi \Delta_{x}^{2}\right)^{-1 / 4} \exp \left[i \bar{p} x / \hbar-(x-\bar{x})^{2} /\left(2 \Delta_{x}\right)^{2}\right] .
$$

Such a state will evolve with time and we need to find whether it will remain a minimum uncertainty state.

An eigenstate $\psi$ of the operator $\hat{a}$, with eigenvalue $\alpha$ will remain an eigenstate if $\partial_{t}[(\hat{a}-\alpha) \psi]=0$. We will seek minimum uncertainty states that have $\Delta_{x}$ and $\Delta_{p}$ constant in time, and hence $\hat{a}$ does not depend on the time. Because $i \hbar \partial_{t} \psi$ $=\hat{H} \psi$, we have $i \hbar \partial_{t}[(\hat{a}-\alpha) \psi]=(\hat{a}-\alpha) \hat{H} \psi-i \hbar \dot{\alpha} \psi=(\hat{a} \hat{H}-\hat{H} \hat{a}$ $-i \hbar \dot{\alpha}) \psi$. For the Hamiltonian $\hat{H}=\hat{p}^{2} / 2 m+V(\hat{x})$,

$$
[\hat{H}, \hat{a}]=-\Delta_{x} \frac{\partial V}{\partial x}-\frac{i \hbar}{2 m \Delta_{x}} \hat{p} .
$$

We require that $\hat{a} \psi=\alpha \psi$ and $[\hat{H}, \hat{a}] \psi=-i \hbar \dot{\alpha} \psi$, and therefore we require $\partial_{x} V \propto x$. This shows that only the harmonic oscillator potential can have minimum uncertainty states with constant spreads. As usual, we write $V=m \omega^{2} x^{2} / 2$, and Eq. (A3) becomes

$$
[\hat{H}, \hat{a}]=-\hbar \omega\left[\frac{1}{2 \Delta_{x}}\left(\frac{\Delta_{x}}{d}\right)^{2} \hat{x}+i \frac{\Delta_{x}}{\hbar}\left(\frac{d}{\Delta_{x}}\right)^{2} \hat{p}\right] .
$$

Therefore, we can maintain $\hat{a} \psi=\alpha \psi$ if we require that $\Delta_{x}$ $=d$. Then, $\hat{a}=\hat{x} / 2 d+i d \hat{p} / \hbar$ and $[\hat{H}, \hat{a}]=-\hbar \omega \hat{a}$. Furthermore $i \hbar \dot{\alpha}=\hbar \omega \alpha$, so $\alpha(t)=\alpha(0) e^{-i \omega t}$. Ehrenfest's theorem shows that $\bar{x}$ and $\bar{p}$ must follow a classical trajectory; thus, we take $\bar{x}=a \cos \omega t, \bar{p}=-m \omega a \sin \omega t$ as the expectation values of position and momentum. Then, $\alpha=\langle\hat{a}\rangle=\bar{x} / 2 d+i d \bar{p} / \hbar$ $=e^{-i \omega t} a / 2 d$.

We have shown that the states for the harmonic oscillator with the minimum uncertainty product will evolve with both $\Delta_{x}$ and $\Delta_{p}$ unchanging if $\Delta_{x}=d$ (and therefore $\Delta_{p}=\hbar / 2 d$ ); that is, they have the same widths as the ground state. These states are known as coherent states.

Note that the operator $\hat{A}=e^{i \omega t} \hat{a}$ has the constant eigenvalue $a / 2 d$. It can be easily seen that $[\hat{H}, \hat{A}]=i \hbar \partial_{t} \hat{A}$, which means that $\hat{A}$ is an invariant operator. ${ }^{12}$ The eigenstates of any invariant operator remain eigenstates (with unchanging eigenvalues) as they evolve.

\section{APPENDIX B: EXPECTATION VALUES FOR THE HALF OSCILLATOR}

The exact expectation values of the position and momentum and their squares are not required for a qualitative understanding of the general behavior of the coherent state, but are needed to calculate details when the wavepacket overlaps the barrier (as shown in Fig. 2). These expectation values can be found by direct integration, but are messy. In what follows, just one integral will be calculated directly, and the other expectation values will be found by other means.

\section{1. $\left\langle\hat{p}^{2}\right\rangle$ AND $\left\langle\hat{x}^{2}\right\rangle$ IN TERMS OF $\langle\hat{H}\rangle$}

The full oscillator coherent state $\psi(x, t)$ is an eigenfunction of $\hat{a}$ with eigenvalue $\alpha=e^{-i \omega t} a / 2 d$. Because $\hat{a}$ is odd, $\psi(-x, t)$ is an eigenfunction of $\hat{a}$ with eigenvalue $-\alpha$. Both $\psi(x, t)$ and $\psi(-x, t)$ are eigenfunctions of $\hat{a}^{2}$ with the same eigenvalue $\alpha^{2}$, and therefore so is the half oscillator coherent state $\phi(x, t)=\psi(x, t)-\psi(-x, t)$. But $\hat{a}^{2}$ can be written as 


$$
\hat{a}^{2}=\frac{1}{2 d^{2}} \hat{x}^{2}-\frac{1}{\hbar \omega} \hat{H}+\frac{i}{2 \hbar}(\hat{x} \hat{p}+\hat{p} \hat{x}),
$$

where $\hat{H}=\hat{p}^{2} / 2 m+m \omega^{2} \hat{x}^{2} / 2$, which ignores the barrier. If we take the real part of the expectation value of Eq. (B1) for a coherent state (of the full or half oscillator), we find

$$
\left\langle\hat{x}^{2}\right\rangle=\frac{2 d^{2}}{\hbar \omega}\langle\hat{H}\rangle+\frac{1}{2} a^{2} \cos 2 \omega t
$$

Because $\langle\hat{H}\rangle$ is a linear combination of $\left\langle\hat{x}^{2}\right\rangle$ and $\left\langle\hat{p}^{2}\right\rangle$, we can solve for the latter; the results can be expressed as

$$
\begin{aligned}
& \langle\hat{V}\rangle=\frac{1}{2}\langle\hat{H}\rangle+\frac{1}{2} m \omega^{2} a^{2} \cos 2 \omega t \\
& \langle\hat{T}\rangle=\frac{1}{2}\langle\hat{H}\rangle-\frac{1}{2} m \omega^{2} a^{2} \cos 2 \omega t,
\end{aligned}
$$

where $\hat{V}=m \omega^{2} \hat{x}^{2} / 2$ and $\hat{T}=\hat{p}^{2} / 2 m$. These results are valid for both the full and the half oscillator; only $\langle\hat{H}\rangle$ differs. Note that the expectation values here are taken over $(-\infty, \infty)$ for the full oscillator, but only over $(0, \infty)$ for the half oscillator.

\section{THE NORMALIZING INTEGRAL}

For the purpose of normalization, the relevant part of the full oscillator coherent state in Eq. (1) is $\tilde{\psi}(x, t)=\exp [i \bar{p} x / \hbar$ $\left.-(x-\bar{x})^{2} / 4 d^{2}\right]$. Simple algebra gives

$$
\begin{aligned}
\widetilde{\phi}(x, t) & =\widetilde{\psi}(x, t)-\widetilde{\psi}(-x, t) \\
& =2 \exp \left[-\frac{1}{4 d^{2}}\left(x^{2}+\bar{x}^{2}\right)\right] \sinh \sigma x,
\end{aligned}
$$

where $\sigma=\left(a / 2 d^{2}\right) e^{-i \omega t}$. Then,

$$
\int_{0}^{\infty}|\widetilde{\phi}(x, t)|^{2} d x=4 \exp \left(-\lambda \bar{x}^{2}\right) I_{\lambda}\left(\sigma, \sigma^{*}\right),
$$

where, with $\lambda=\left(2 d^{2}\right)^{-1}$,

$$
\begin{aligned}
I_{\lambda}(\sigma, \rho) & =\int_{0}^{\infty} e^{-\lambda x^{2}} \sinh \sigma x \sinh \rho x d x \\
& =\frac{1}{4} \sqrt{\frac{\pi}{\lambda}}\left[e^{(\sigma+\rho)^{2} / 4 \lambda}-e^{(\sigma-\rho)^{2} / 4 \lambda}\right] .
\end{aligned}
$$

The integral may be evaluated using $2 \sinh \sigma x \sinh \rho x$ $=\cosh (\sigma+\rho) x-\cosh (\sigma-\rho) x$, which reduces the problem to evaluating $\int_{0}^{\infty} e^{-\lambda x^{2}} \cosh \gamma x d x$. This integration can be extended to $(-\infty, \infty)$, because the integrand is even. We then express cosh $\gamma x$ in terms of exponentials and complete the square in the combined exponent as usual.

If we substitute $\sigma=\left(a / 2 d^{2}\right) e^{-i \omega t}, \rho=\sigma^{*}$, and $\lambda=\left(2 d^{2}\right)^{-1}$ into Eq. (B6), we obtain $\int_{0}^{\infty}|\widetilde{\phi}|^{2} d x=\sqrt{2 \pi} d\left[1-\exp \left(-a^{2} / 2 d^{2}\right)\right]$. Hence, using $\int_{-\infty}^{\infty}|\widetilde{\psi}|^{2} d x=\sqrt{2 \pi} d$, the extra normalization required for $\widetilde{\phi}$ [and for $\phi$ in Eq. (2)] is $N_{1}=1-\exp \left(-a^{2} / 2 d^{2}\right)$.

It is necessary that $N_{1} \approx 1$ when $a \gg d$. When $\psi(x, t)$ is almost fully in $x>0$, then $\psi(-x, t)$ has only negligible presence in $x>0$, and $\phi(x, t) \approx \psi(x, t)$ throughout $x>0$. Therefore, if $\psi(x, t)$ is normalized, then so will be $\phi(x, t)$.

\section{EXPECTATION VALUES OF $\hat{\boldsymbol{x}}^{2}$ AND $\hat{\boldsymbol{H}}$}

Because $\quad \partial I_{\lambda} / \partial \lambda=-\int_{0}^{\infty} x^{2} \exp \left(-\lambda x^{2}\right) \sinh \sigma x \sinh \rho x d x$, it follows that $\left\langle\hat{x}^{2}\right\rangle=\left(-\partial I_{\lambda} / \partial \lambda\right) / I_{\lambda}=1 / 2 \lambda+\left[\sigma^{2}+\rho^{2}\right.$ $+2 \sigma \rho \operatorname{coth}(\sigma \rho / 2 \lambda)] / 4 \lambda^{2}$. If we insert the values for $\sigma, \rho$, and $\lambda$, we obtain

$$
\left\langle\hat{x}^{2}\right\rangle=d^{2}+\frac{1}{2} a^{2} \operatorname{coth} \frac{a^{2}}{4 d^{2}}+\frac{1}{2} a^{2} \cos 2 \omega t .
$$

The comparison of Eq. (B7) with Eq. (B2) leads to

$$
\langle\hat{H}\rangle=\frac{1}{2} \hbar \omega\left(1+\frac{1}{2} \frac{a^{2}}{d^{2}} \operatorname{coth} \frac{a^{2}}{4 d^{2}}\right) .
$$

For the full oscillator $\hat{H}=\hbar \omega\left(\hat{a}^{\dagger} \hat{a}+1 / 2\right)$, and thus $\langle\hat{H}\rangle$ $=(\hbar \omega / 2)\left(1+a^{2} / 2 d^{2}\right)$ for the coherent state. For $a \gg d$, this expression for $\langle\hat{H}\rangle$ is approached by the value in Eq. (B8), as expected.

\section{EXPECTATION VALUES OF $\hat{\boldsymbol{x}}$ AND $\hat{\boldsymbol{p}}$}

To calculate $\langle\hat{x}\rangle$ and $\langle\hat{p}\rangle$, we need to find the effect of the barrier potential $W(x)$, where $W(x)=0$ for $x>0$ and $W(x)$ $=\infty$ for $x \leq 0$. Then the evolution equations for the expectation values for any wavepacket are $m d_{t}\langle\hat{x}\rangle=\langle\hat{p}\rangle$ and $d_{t}\langle\hat{p}\rangle$ $=-m \omega^{2}\langle\hat{x}\rangle-\left\langle\partial_{x} \hat{W}\right\rangle$. Any contributions to $\left\langle\partial_{x} \hat{W}\right\rangle$ must come from the neighborhood of $x=0$. To determine these contributions, multiply Schrödinger's equation for $\phi$ by $\partial_{x} \phi^{*}$, add the complex conjugate equation, and integrate each term of the resulting equation over an infinitesimally narrow strip $(-\epsilon, \epsilon)$ about the barrier. The only singular terms in Schrödinger's equation are the term involving $\partial_{x}^{2} \phi$, which is singular because $\phi$ has a discontinuous derivative at the barrier, and the term involving $W$. The first term gives a contribution $-\left(\hbar^{2} / 2 m\right)\left(\partial_{x} \phi^{*} \partial_{x}^{2} \phi+\partial_{x} \phi \partial_{x}^{2} \phi^{*}\right)=-\left(\hbar^{2} / 2 m\right) \partial_{x}\left(\left|\partial_{x} \phi\right|^{2}\right)$, which integrates to $-\left(\hbar^{2} / 2 m\right)\left|\partial_{x} \phi\right|_{x=\epsilon}^{2}$. The other singular term is $W(x) \partial_{x}\left(\phi^{*} \phi\right)$, which integrates by parts to $\left[W(x)|\phi|^{2}\right]_{-\epsilon}^{\epsilon}-\int_{-\epsilon}^{\epsilon}|\phi|^{2} \partial_{x} W(x) d x$. The first of these terms is zero, and we conclude that

$$
\left\langle-\partial_{x} W\right\rangle=\frac{\hbar^{2}}{2 m}\left|\partial_{x} \phi\right|_{x=0}^{2} .
$$

Therefore, the evolution equations are $m d_{t}\langle\hat{x}\rangle=\langle\hat{p}\rangle$ and $d_{t}\langle\hat{p}\rangle=-m \omega^{2}\langle\hat{x}\rangle+f(t)$ with $f(t)=\left(\hbar^{2} / 2 m\right)\left|\partial_{x} \phi\right|_{x=0}^{2}$. To solve these equations, it is helpful to combine them into $d_{t}\langle\hat{a}\rangle$ $=-i \omega\langle\hat{a}\rangle+i(d / \hbar) f(t)$; hence, $d_{t}\left(e^{i \omega t}\langle\hat{a}\rangle\right)=i(d / \hbar) e^{i \omega t} f(t)$, and

$$
\langle\hat{a}\rangle=e^{-i \omega t}\langle\hat{a}\rangle_{t=0}+i(d / \hbar) e^{-i \omega t} \int_{0}^{t} e^{i \omega \tau} f(\tau) d \tau .
$$

The real and imaginary parts of $\langle\hat{a}\rangle$ give $\langle\hat{x}\rangle$ and $\langle\hat{p}\rangle$ valid for any wavepacket.

For the half oscillator coherent state, $\left|\partial_{x} \phi\right|_{x=0}^{2}$ $=N_{1}^{-1} N_{0}^{-1} a^{2} d^{-4} \exp \left(-\bar{x}^{2} / 2 d^{2}\right)$, from Eq. (B4), and therefore 


$$
\begin{aligned}
\langle\hat{a}\rangle= & N_{1}^{-1} \alpha\left[\operatorname{erf}\left(\frac{a}{\sqrt{2} d} \cos \omega t\right)\right. \\
& \left.+i e^{-a^{2} / 2 d^{2}} \operatorname{erfi}\left(\frac{a}{\sqrt{2} d} \sin \omega t\right)\right],
\end{aligned}
$$

where $\alpha=\bar{x} / 2 d+i d \bar{p} / \hbar=e^{-i \omega t} a / 2 d, \operatorname{erf}(z)=(2 / \sqrt{\pi}) \int_{0}^{z} e^{-u^{2}} d u$ is the error function, and $\operatorname{erfi}(z)=(2 / \sqrt{\pi}) \int_{0}^{z} e^{u^{2}} d u$, so that $\operatorname{erf}(i z)=i \operatorname{erfi}(z)$. Because $\langle\hat{a}\rangle=\langle\hat{x}\rangle / 2 d+i d\langle\hat{p}\rangle / \hbar$, Eq. (B11) can be used to derive

$$
\begin{aligned}
\langle\hat{x}\rangle= & N_{1}^{-1}\left[\bar{x} \operatorname{erf}\left(\frac{\bar{x}}{\sqrt{2} d}\right)-\frac{2 d^{2}}{\hbar} \bar{p} e^{-a^{2} / 2 d^{2}}\right. \\
& \left.\times \operatorname{erfi}\left(-\frac{\sqrt{2} d}{\hbar} \bar{p}\right)\right], \\
\langle\hat{p}\rangle= & N_{1}^{-1}\left[\bar{p} \operatorname{erf}\left(\frac{\bar{x}}{\sqrt{2} d}\right)+\frac{\hbar}{2 d^{2}} \bar{x} e^{-a^{2} / 2 d^{2}}\right. \\
& \left.\times \operatorname{erfi}\left(-\frac{\sqrt{2} d}{\hbar} \bar{p}\right)\right] .
\end{aligned}
$$

These results were used to generate Fig. 2. When the wavepacket is well clear of the barrier, that is, $\bar{x} \gg d$, then $N_{1}$ $\approx 1, \operatorname{erf}(\bar{x} / \sqrt{2} d) \approx 1$, and the second term is negligibly small in Eq. (B12). Then, $\langle\hat{x}\rangle \approx \bar{x}$ and $\langle\hat{p}\rangle \approx \bar{p}$, as expected.

The integral in Eq. (B10) can be carried out by substituting $u=(a / \sqrt{2} d) \cos \omega \tau$ into $\int_{0}^{t} \sin \omega \tau \exp \left(-\left(a^{2} / d^{2}\right)\right.$ $\left.\cos ^{2} \omega \tau\right) d \tau$ to give an integral with the form of an error function, and by writing $\int_{0}^{t} \cos \omega \tau \exp \left(-\left(a^{2} / d^{2}\right) \cos ^{2} \omega \tau\right) d \tau$ as $e^{-a^{2} / 2 d^{2}} \int_{0}^{t} \sin \omega \tau \exp \left(\left(a^{2} / d^{2}\right) \sin ^{2} \omega \tau\right) d \tau$, which can be expressed as an erfi function by the substitution $v=(a / \sqrt{2} d)$ $\sin \omega \tau$.

\section{RELATION OF $\left\langle\hat{p}^{2}\right\rangle$ AND $\left\langle\hat{x}^{2}\right\rangle$ TO $\langle\hat{H}\rangle$ FOR AN ARBITRARY WAVEPACKET}

Because the barrier affects the evolution equations for $\langle\hat{x}\rangle$ and $\langle\hat{p}\rangle$, does it also enter the evolution equations for $\left\langle\hat{x}^{2}\right\rangle$ and $\left\langle\hat{p}^{2}\right\rangle$ ? It will now be shown that the barrier does not change the form of these equations (although it does affect the value of $\langle\hat{H}\rangle$ ), and relations similar to Eq. (B3) hold for any wavepacket.

Including the barrier potential $W$, the evolution equations are $m d_{t}\left\langle\hat{x}^{2}\right\rangle=2\langle\hat{\chi}\rangle$, where $\hat{\chi}=(\hat{x} \hat{p}+\hat{p} \hat{x}) / 2$, and $m d_{t}\langle\hat{\chi}\rangle$ $=-m \omega^{2}\left\langle\hat{x}^{2}\right\rangle+\left\langle\hat{p}^{2}\right\rangle / m-\left\langle\hat{x} \partial_{x} W\right\rangle$. Reasoning similar to that used for $\langle\hat{p}\rangle$ shows that the last term must be zero. [Multiply
Schrödinger's equation for $\phi$ by $x \partial_{x} \phi^{*}$ and add the complex conjugate. Integrating over the term involving $\partial_{x}^{2} \phi$ leads to $\left.\left.\int_{-\epsilon}^{\epsilon} x \partial_{x}\left(\partial_{x} \phi^{*} \partial_{x} \phi\right) d x=x \mid \partial_{x} \phi\right)\left.\left.\right|^{2}\right|_{-\epsilon} ^{\epsilon}-\int_{-\epsilon}^{\epsilon} \mid \partial_{x} \phi\right)\left.\right|^{2} d x$, which clearly goes to zero as $\epsilon \rightarrow 0$ and similarly for the term in $\partial_{x} W$. A similar argument, after multiplying Schrödinger's equation by $\phi^{*}$, shows that $\langle W\rangle$ is zero.]

Therefore, $m d_{t}\left\langle\hat{x}^{2}\right\rangle=2\langle\hat{\chi}\rangle$ and $m d_{t}\langle\hat{\chi}\rangle=2\langle\hat{H}\rangle-2 m \omega^{2}\left\langle\hat{x}^{2}\right\rangle$. Because $\langle\hat{H}\rangle$ is constant, these equations can be solved to give $m \omega^{2}\left\langle\hat{x}^{2}\right\rangle=\langle\hat{H}\rangle+A \cos 2 \omega\left(t-t_{0}\right)$, where $A$ and $t_{0}$ are arbitrary constants. Therefore,

$$
\begin{aligned}
& \langle\hat{V}\rangle=\frac{1}{2}\langle\hat{H}\rangle+\frac{1}{2} A \cos 2 \omega\left(t-t_{0}\right), \\
& \langle\hat{T}\rangle=\frac{1}{2}\langle\hat{H}\rangle-\frac{1}{2} A \cos 2 \omega\left(t-t_{0}\right),
\end{aligned}
$$

which is similar to Eq. (B3), but now valid for any wavepacket of the half oscillator.

This type of analysis of the evolution of expectation values can be done where there is a repulsive barrier together with any potential that is at most quadratic in $x$. The potential must be at most quadratic so that $\langle F(x)\rangle=F(\langle x\rangle)$, where $F(x)=-\partial_{x} V(x)$ is the force.

${ }^{a)}$ Electronic mail: mark.andrews@anu.edu.au

${ }^{1}$ Eugen Merzbacher, Quantum Mechanics (John Wiley \& Sons, New York, 1998), 3rd ed., Sec. 10.7.

${ }^{2}$ Kurt Gottfried and Tung-Mow Yan, Quantum Mechanics: Fundamentals (Springer, New York, 2003) 2nd ed., p. 181.

${ }^{3}$ S. Howard and S. K. Roy, "Coherent states of a harmonic oscillator," Am. J. Phys. 55, 1109-1117 (1987).

${ }^{4} \mathrm{H}$. A. Gersch, "Time evolution of minimum uncertainty states of a harmonic oscillator," Am. J. Phys. 60, 1024-1030 (1992).

${ }^{5}$ Richard R. Robinett, Quantum Mechanics (Oxford University Press, New York, 1997), p. 185.

${ }^{6}$ Nouredine Zettili, Quantum Mechanics (John Wiley \& Sons, New York, 2001), p. 253.

${ }^{7}$ All states of the half oscillator must approach zero at the barrier. All odd eigenstates of the full oscillator will provide eigenstates of the half oscillator and there can be no others. If $\psi(x)$ is any eigenfunction of the half oscillator for $x>0$, then extending this function to negative $x$ by defining $\psi(-x)=-\psi(x)$ will give an odd eigenfunction of the full oscillator.

${ }^{8}$ Mark Andrews, "Wave packets bouncing bouncing off walls," Am. J. Phys. 66, 252-254 (1998).

${ }^{9}$ M. A. Doncheskia and R. W. Robinett, "Expectation value analysis of wave packet solutions for the quantum bouncer: Short-term classical and long-term revival behaviors," Am. J. Phys. 69, 1084-1090 (2001), and references therein.

${ }^{10}$ William H. Mather and Ronald F. Fox, "Coherent-state analysis of the quantum bouncing ball,” Phys. Rev. A 73, 032109-1-9 (2006).

${ }^{11}$ Reference 1, Sec. 10.5.

${ }^{12}$ Mark Andrews, "Total time derivatives of operators in elementary quantum mechanics," Am. J. Phys. 71, 326-332 (2003). 